\title{
ON THE INTRINSIC FORM FOR SECOND ORDER DIFFERENTIAL OPERATORS
}

\author{
Dedicated to Paul Lévy \\ on the occasion of his seventieth birthday \\ BY \\ William Feller ${ }^{1}$
}

\section{Introduction}

A function $f$ will be said to be in the local domain of the linear operator $\mathfrak{A}$ at the point $s$, (in symbols: $f \in D(\mathfrak{A}, s)$ ), if $f$ and $\mathfrak{A} f$ are defined and continuous in some neighborhood of $s$. Similarly, the domain $D(\mathfrak{A}, I)$ of $\mathfrak{A}$ for the interval $I$ consists of all $f$ such that $f$ and $\mathfrak{U} f$ are continuous in $I$.

The differential operator

$$
\mathfrak{A}=a D_{s}^{2}+b D_{s}+c, \quad a>0, \quad D_{s}=\frac{d}{d s}
$$

enjoys the following obvious properties:

(1) Local character: If $f(s)=0$ for all $s$ in a neighborhood of the point $s_{0}$, then $f \in D\left(\mathfrak{A}, s_{0}\right)$ and $\mathfrak{A} f\left(s_{0}\right)=0$.

(2) $\mathfrak{A}$ is nontrivial: To each point $s$ of the interval of definition there exists an $f \in D(\mathfrak{R}, s)$ such that $f(a) \neq 0$ and $\mathfrak{A} f(s) \neq 0$.

(3) Weak minimum property: Let $f \in D(\mathfrak{A}, s)$ be nonnegative in a neighborhood of $s$ and $f(s)=0$. Then $\mathfrak{A} f(s) \geqq 0$.

In other words, if the point $s$ is both a zero and a local minimum for $f$, then $\mathfrak{A} f(s) \geqq 0$. For the pure differential operator $\mathfrak{U}=a D_{s}^{2}+b D_{s}$ (where $a>0$ ) the property (3) may be sharpened to

$\left(3^{\prime}\right)$ Strong minimum property: If $f \in D(\mathfrak{A}, s)$ has a local minimum at $s$, then $\mathfrak{Y} f(s) \geqq 0$.

Various problems have led the author to derive the general form of linear operators in one dimension having these properties [1]. The class of such operators forms a natural generalization of the classical second order differential operators. It has been shown elsewhere, [1], [2], [3], that their use has considerable advantages. The use of the new canonical form renders the theory more satisfactory and at the same time simpler; it achieves an unexpected unification and is more adapted for many applied problems.

Received June 26, 1957.

1 This research was done while the author was on sabbatical leave of absence with the support of the United States Air Force through the Air Force Office of Scientific Research of the Air Research and Development Command. 
It is the purpose of this paper to present an improved derivation of a canonical form of operators with the properties (1), (2), (3) and to provide additional information on various points.

The problem may be viewed under two different aspects. It is easily shown that the equation $\mathfrak{A} u=0$ admits of at most two independent solutions (provided independence is defined as in footnote 3). For certain purposes it is natural to postulate the existence (at least locally) of two independent solutions, and the general form of our operators can then be derived with ease. If $\mathfrak{U}$ has the strong minimum property $\left(3^{\prime}\right)$, one of the two solutions may be taken as the constant function, and the other is automatically strictly monotonic. It may be used to reparametrize the interval of definition in such a way that the general solution of $\mathfrak{A} u=0$ is a linear function.

The crucial Section 3 contains the derivation of a canonical form $D_{m} D_{x}$ of our operators with this additional property; Section 4 reduces the general case of operators with the strong minimum property and two independent solutions to the simple case of Section 3. The analogous problems for operators with the weak minimum property are treated in Sections 7 and 8 leading to the canonical representations $(8.1)$ and $\left(^{*}\right)$. These four sections are independent of the remainder of the paper.

Unfortunately, when only the properties (1), (2), (3) (or $\left(3^{\prime}\right)$ ) are postulated, the equation $\mathfrak{A} u=0$ need not have any solutions. The nonexistence may be due to two conditions:

(a) The original operator $\mathfrak{U}$ may be defined on too small a domain.

(b) The operator $\mathfrak{A}$ behaves essentially as a first order operator (the case of a vanishing coefficient $a$ in (1.1)).

In case (a) the definition of $\mathfrak{A}$ may be extended, without violating the postulated properties (1), (2), (3), in such a way that the preceding theory applies to the extended operator. This is the less elegant part of the derivation and is contained in Sections 5 and 9, respectively, for operators with the strong and weak minimum property.

The singular (or first order) points at which the $\mathfrak{A}$ degenerates into a first order operator are described intrinsically in Definition 2.2, and are treated in Section 6 . They may, but need not, be isolated points, but the regular points always form an open set.

Section 10 contains three illustrative examples.

\section{The operator $D_{m} D_{x}$}

In a fixed interval $I:-\infty \leqq x_{-}<x<x_{+} \leqq \infty$ let $m$ be a strictly increasing function continuous on the right:

$$
m \uparrow \quad m(x)=\lim _{h \downarrow 0} m(x+h) ;
$$

it is not required that $m$ be bounded at either end. The difference $m(y)-m(x)$ (where $x<y$ ) should be thought of as a measure of the half-open interval $(x, y]$. 
If $m$ is continuous, the interval $I$ may be parametrized by $m$ instead of $x$, and differentiation with respect to $m$ is in this case defined in the familiar fashion. For a discontinuous $m$ we adopt the same definition with the agreement to consider increments only over closed intervals. Thus, if $g$ is a function defined in $I$ such that the right and left limits $g(x+)$ and $g(x-)$ exist for each $x \in I$, we define the right derivative of $g$ with respect to $m$ by

$$
D_{m}^{+} g(x)=\lim _{y \downarrow x} \frac{g(y+)-g(x-)}{m(y+)-m(x-)},
$$

provided, of course, the limit exists. The left derivative $D_{m}^{-} g$ is defined symmetrically. Clearly at a point of discontinuity of $m$

$$
D_{m}^{+} g(x)=D_{m}^{-} g(x)=\frac{g(x+)-g(x-)}{m(x+)-m(x-)},
$$

and a differentiable $g$ is continuous at each point of continuity of $m$.

We now define an operator which will prove to be the natural generalization. of $a D_{s}^{2}+b D_{s}$.

Definition 2.1. We say that a function $f$ is in the domain $D(\mathfrak{A}, I)$ of the operator $\mathfrak{A}=D_{m} D_{x}$ in the interval $I$ if:

(i) The right and left derivatives $f^{+}=D_{x}^{+} f$ and $f^{-}=D_{x}^{-} f$ exist and

$$
\begin{aligned}
& f^{+}(x)=\lim _{y \downarrow x} f^{+}(y)=\lim _{y \downarrow x} f^{-}(y) \\
& f^{-}(x)=\lim _{y \uparrow x} f^{+}(y)=\lim _{y \uparrow x} f^{-}(y)
\end{aligned}
$$

for each $x \in I$.

(ii) $D_{m} D_{x}^{+} f$ exists and is continuous in $I$.

The definition (2.2) implies that $D_{m} D_{x}^{+} f$ is identical with $D_{m} D_{x}^{-} f$ and we shall write

$$
\mathfrak{U} f=D_{m} D_{x} f=D_{m} D_{x}^{-} f=D_{m} D_{x}^{+} f .
$$

Clearly our definition applies equally to any subinterval of $I$.

Lemma 2.1. The operator $\mathfrak{A}=D_{m} D_{x}$ has the properties (1), (2), (3').

Proof. Only $\left(3^{\prime}\right)$ requires comment. Suppose that $f \in D(\mathfrak{A}, x)$ has a local minimum at the point $x$. Then $f^{-}(x) \leqq 0$ and $f^{+}(x) \geqq 0$. It follows now from (2.4) that $f^{+}$cannot strictly decrease in any neighborhood $N$ of $x$ as would be the case if $\mathfrak{A} f<0$ in $N$.

The converse of this lemma is true in the sense that the operators of the form $\mathfrak{A}=D_{m} D_{x}$ are essentially the only ones ${ }^{2}$ with the properties (1), (2), $\left(3^{\prime}\right)$. An exception occurs only in connection with singular points, where

2 To reduce $a D_{s}^{2}+b D_{s}$ to the canonical form $D_{m} D_{x}$, choose for $x$ an increasing solution of $a D_{s}^{2} x+b D_{s} x=0$. Then $a D_{s}^{2}+b D_{s}=\alpha D_{x}^{2}$, where $\alpha>0$ if $a>0$. Now put $m=$ $\int \alpha^{-1} d x$. 
$\mathfrak{U}$ has the character of a first order differential operator. To give an intrinsic characterization of such points note that a function $f$ in the domain of $\mathfrak{A}$ has strong regularity properties and, in particular, cannot oscillate widely. More precisely, we have

Lemma 2.2. Let $\mathfrak{A}$ have properties (1), (2), (3'). Suppose that $f \in D\left(\mathfrak{A}, x_{0}\right)$ and $\mathfrak{A} f\left(x_{0}\right)>0$. Then there exist one-sided neighborhoods $x_{0} \leqq x<x_{0}+\delta$ and $x_{0}-\delta<x \leqq x_{0}$ in each of which $f$ is strictly monotonic.

Proof. Assume the assertion to be false, that is, suppose that there exists a sequence of points $x_{n} \rightarrow x_{0}$ such that $f$ has a local maximum at $x_{2 n}$ and a local minimum at $x_{2 n+1}$. By the strong minimum property $\left(3^{\prime}\right)$ we have $\mathfrak{A} f\left(x_{2 n}\right) \leqq 0$ and $\mathfrak{A} f\left(x_{2 n+1}\right) \geqq 0$, and the assumed continuity of $\mathfrak{A} f$ requires therefore that $\mathfrak{Y} f\left(x_{0}\right)=0$, against hypothesis.

According to this lemma the relation $\mathfrak{A} f\left(x_{0}\right)>0$ implies that either $f$ is strictly monotonic in some neighborhood of $x_{0}$, or $f$ has a local minimum at $x_{0}$. In the special case of a first order differential operator $\mathfrak{A}=b D_{x}$ with $b>0$, the relation $\mathfrak{A} f\left(x_{0}\right)>0$ implies that $f$ is increasing, but for an honest second order operator $\mathfrak{A} f\left(x_{0}\right)>0$ is possible both for increasing and for decreasing $f$. These remarks will explain the

Definition 2.2. Let $\mathfrak{A}$ have the properties (1), (2), (3'). The point $x_{0}$ is regular for $\mathfrak{A}$ if $D\left(\mathfrak{H}, x_{0}\right)$ contains both an increasing and a decreasing $f$ such that $\mathfrak{A} f\left(x_{0}\right)>0$.

A point which is not regular is called a first order point.

Theorem 6.1 shows that at first order points $\mathfrak{A}$ reduces to a first derivative. In the absence of first order points $\mathfrak{A}$ is of the nature of a second order differential operator. This is seen from the following two basic theorems concerning operators with the strong minimum property $\left(3^{\prime}\right)$.

Theorem 2.1. Suppose that in an interval $I: s_{-}<s<s_{+}$the operator $\mathfrak{A}$ has the properties (1), (2), (3') and that all points of $I$ are regular.

There exist in $I$ a strictly increasing continuous function $x$ and a strictly increasing right-continuous function $m$ such that $\mathfrak{A} f=D_{m} D_{x} f$ for each $f$ in a local domain of $\mathfrak{A}$.

The variable $x$ may be used as scale parameter for $I$ (so that $I: x_{-}<x<x_{+}$) and is determined uniquely up to a trivial linear transformation $x \rightarrow p x+$ const. For given $x$ the "canonical measure" $m$ is determined up to an irrelevant additive constant.

Note that $\mathfrak{A}$ may be defined on a small domain whence $D_{m} D_{x}$ may be a proper extension of $\mathfrak{A}$.

A different aspect of the situation is covered by

THEOREM 2.2. The assertions of the preceding theorem remain valid if the condition that all points be regular is replaced by the following: 
To each $s \in I$ there corresponds a neighborhood $N_{s}$ of $s$ such that in $N_{s}$ there exist two linearly independent ${ }^{3}$ solutions $u_{i}$ of $\mathfrak{A} u_{i}=0$.

\section{A special case}

We shall prove the most interesting special case of Theorem 2.1 directly and then reduce the general case to it.

Theorem 3.1. Suppose that $\mathfrak{A}$ is defined in the interval $I:-\infty \leqq x_{-}<$ $x<x_{+} \leqq \infty$ and has properties (1), (2), (3'). Suppose furthermore that ${ }^{4}$

$$
\mathfrak{U} \mathbf{1}=0, \quad \mathfrak{Y} \mathbf{x}=0 .
$$

Then there exists in $I$ a strictly increasing right-continuous $m$ such that $\mathfrak{A} f=D_{m} D_{x} f$ for each $f$ in a domain of $\mathfrak{A}$. This $m$ is determined up to an irrelevant additive constant.

The proof will be based on the following

LEMma 3.1. Adhering to the conditions of the preceding theorem, let $\mathfrak{A} f$ be continuous and strictly positive. Then $f$ is convex downward.

Proof. Let $p$ and $q$ be constants, and define $u$ by $u(x)=f(x)-p x-q$. Then $\mathfrak{A} u=\mathfrak{A} f>0$ so that $u$ can have no local maximum in $I$. If $x_{1}<x_{2}$ are two zeros of $u$, then $u$ must be strictly negative in the interval $x_{1}<x<x_{2}$ and hence the graph of $f$ lies below each of its chords.

Proof of Theorem 3.1. By property (2) there exists a function $g$ such that $\mathfrak{A} g>0$ in some neighborhood of an arbitrarily prescribed point $x_{0}$. In this neighborhood $g$ is convex. An arbitrary $f \in D\left(\mathfrak{A}, x_{0}\right)$ may be represented as a difference, $f=(f+t g)-t g=f_{1}-f_{2}$ of two functions $f_{i}$, and choosing $t$ sufficiently large we shall have $\mathfrak{A} f_{i}>0$ in some neighborhood $N$ of $x_{0}$. It follows that $f_{i}$ is convex in $N$, and hence the one-sided derivatives $f^{+}$and $f^{-}$ exist everywhere in $N$ and satisfy the continuity requirement (2.4). Since $x_{0}$ is arbitrary, it follows that $f^{+}$and $f^{-}$exist and satisfy (2.4) for each $x \in I$ and $f \in D(\mathfrak{A}, I)$.

Suppose now that $\mathfrak{A} f=\phi$ and $\mathfrak{A} g=\gamma$ are continuous and positive in the interval $I_{0} \subset I$. Then

$$
\mu(x)=g^{+}(x)
$$

defines a strictly increasing right-continuous function $\mu$ defined in $I_{0}$.

Let $t$ be a real parameter and consider the function

$$
u_{t}=f-t g \text {. }
$$

3 Two functions $u_{i}$ are called independent in an interval $I_{0}$ if a nontrivial relationship $p_{1} u_{1}+p_{2} u_{2}=0$ does not exist in any subinterval of $I_{0}$. See example (c) of Section 10 concerning this convention.

${ }^{4}$ We denote by bold face $\mathrm{x}$ the function whose value equals $x$ for each $x$, and by 1 the function which equals 1 everywhere. No new symbol was thought necessary for the zero function. 


$$
t<\min _{x \in I_{0}} \frac{\phi(x)}{\gamma(x)},
$$

then $\mathfrak{A} u_{t}$ is strictly positive in $I_{0}$, and the convexity of $u_{t}$ implies that its right derivative increases. In other words, (3.4) implies that

$$
f^{+}\left(\xi_{2}\right)-f^{+}\left(\xi_{1}\right)>t\left\{\mu\left(\xi_{2}\right)-\mu\left(\xi_{1}+\right)\right\}, \quad \xi_{1}<\xi_{2} .
$$

If $t>\max \phi(x) / \gamma(x)$, the inequality in (3.5) is reversed. Thus the increment of $f^{+}$is an interval function with the mean value property

$$
\begin{aligned}
\min _{\xi_{1} \leqq x \leqq \xi_{2}} \frac{\phi(x)}{\gamma(x)} \cdot\left\{\mu\left(\xi_{2}\right)-\mu\left(\xi_{1}+\right)\right\} & \leqq f^{+}\left(\xi_{2}\right)-f^{+}\left(\xi_{1}+\right) \\
& \leqq \max _{\xi_{1} \leqq x \leqq \xi_{2}} \frac{\phi(x)}{\gamma(x)} \cdot\left\{\mu\left(\xi_{2}\right)-\mu\left(\xi_{1}+\right)\right\} .
\end{aligned}
$$

This being the property on which the construction of the Riemann-Stieltjes integral is based, we conclude that

$$
f^{+}\left(\xi_{2}\right)-f^{+}\left(\xi_{1}+\right)=\int_{\xi_{1}+}^{\xi_{2}+} \frac{\phi(x)}{\gamma(x)} d \mu(x)
$$

Finally, putting

(3.7) takes on the form

$$
m=\int \frac{d \mu}{\gamma}
$$

$$
f^{+}\left(\xi_{2}\right)-f^{+}\left(\xi_{1}+\right)=\int_{\xi_{1}+}^{\xi_{2}+} \mathfrak{A} f \cdot d m .
$$

This is the integrated version of $\mathfrak{A} f=D_{m} D_{x} f$, and we have proved Theorem 3.1 locally: to each $x \in I$ there exists a neighborhood $N_{x}$ such that in it $\mathfrak{A} f=D_{m} D_{x} f$ for each $f$ in the domain of $\mathfrak{A}$. A glance at (3.9) shows that the weight function $m$ is determined up to an irrelevant additive constant.

To prove the theorem in the large, choose among these $N_{x}$ denumerably many, say $I_{k}, k=0, \pm 1, \pm 2, \cdots$, which cover the interval $I$ and such that $I_{k}$ has an overlap with $I_{k-1}$ and $I_{k+1}$, but no other interval $I_{k}$. It is then clear that, starting from $I_{0}$, the free additive constants in $I_{1}, I_{-1}, I_{2}, I_{-2}, \cdots$ can be adjusted so that the definitions of $m$ agree in the common parts $I_{k} \cap I_{k+1}$, and Theorem 3.1 is thus proved.

\section{Proof of Theorem 2.2}

In this section $\mathfrak{A}$ is assumed to have properties (1), (2), (3') and to be defined in the interval $I: s_{-}<s<s_{+}$. We shall reduce Theorem 2.2 to Theorem 3.1.

Lemma 4.1. To each $s \in I$ there corresponds a neighborhood $N_{s}$ with the following property: If $u$ is a function such that $\mathfrak{A} u=0$ in $N_{s}$ and $u\left(s_{1}\right)=u\left(s_{2}\right)$ for two points $s_{1}<s_{2}$ in $N_{s}$, then $u=$ const. in the interval $\left(s_{1}, s_{2}\right)$.

Proof. We take for $N_{s}$ any interval around $s$ for which there exists an $f$ 
such that $\mathfrak{A} f>0$ in $N_{s}$. Property (2) guarantees the existence of such a neighborhood. Suppose the assertion false, e.g. that $u\left(s_{1}\right)=u\left(s_{2}\right)<u\left(s_{0}\right)-\varepsilon$ for some $s_{0}$ such that $s_{1}<s_{0}<s_{2}$ and $\varepsilon>0$. Put for abbreviation $F=f+\nu \varepsilon^{-1} u$, where $\nu$ is a constant exceeding the oscillation of $f$ in $N_{s}$. Then $F\left(s_{0}\right)>F\left(s_{i}\right)$ for $i=1,2$, and hence the maximum of $F$ in $\left(s_{1}, s_{2}\right)$ is attained at an interior point although $\mathfrak{A} F=\mathfrak{X} f>0$. This contradicts the property $\left(3^{\prime}\right)$.

Lemm 4.2. If $\mathbf{1}$ is in the domain of $\mathfrak{A}$, then $\mathfrak{A} \mathbf{1}=0$. Otherwise $\mathfrak{A}$ may be extended by defining $\mathfrak{Y} \mathbf{1}=0$, and the thus extended operator has again the properties (1), (2), $\left(3^{\prime}\right)$.

Proof. 'The functions $\mathbf{1}$ and $\mathbf{- 1}$ have a local minimum at each point, and the minimum property $\left(3^{\prime}\right)$ therefore requires that $\mathfrak{Y} \mathbf{1}=0$. If $\mathbf{1}$ is not in the domain $D(\mathfrak{A}, s)$, let $D^{\prime}$ be the set of all functions of the form $f+c \mathbb{1}$ where $f \epsilon D(\mathfrak{A}, s)$ and $c$ is a constant. The representation $f+c \mathbf{1}$ is obviously unique, and $f+c 1$ has extrema at the same places as $f$. It is therefore possible to put $\mathfrak{A}(f+c \mathbf{1})=\mathfrak{A} f$ without violating any of the three requirements $(1),(2),\left(3^{\prime}\right)$.

Inmma 4.3. Suppose that to each $s \in I$ there corresponds a neighborhood $N_{s}^{\prime}$ of $s$ such that in $N_{s}^{\prime}$ there exist two independent solutions $u_{i}$ of $\mathfrak{A} u_{i}=0$.

Then there exists in $I$ a strictly increasing continuous $x$ such that $\mathfrak{A} x=0$. Furthermore $\mathfrak{H} \mathbf{1}=0 . \quad$ If in some interval $I^{\prime} \subset I$ the function $u$ satisfies $\mathfrak{A} u=0$, then $u=p x+q 1$ where $p$ and $q$ are constant.

Proof. In the intersection $N_{s} \cap N_{s}^{\prime}$ the functions $u_{i}$ are monotonic in the weak sense by virtue of Lemma 4.1. If need be, let us extend $\mathfrak{A}$ in accordance with the preceding lemma by setting $\mathfrak{Y} 1=0$. Let $s_{1}<s_{2}$ be two arbitrary points of $N_{s} \cap N_{s}^{\prime}$, and put $v_{i}=u_{\imath}-u_{\imath}\left(s_{1}\right) 1$. Then (using the letter $\mathfrak{A}$ for the extended operator) $\mathfrak{A} v_{i}=0$, and hence $v=v_{2}\left(s_{2}\right) v_{1}-v_{1}\left(s_{2}\right) v_{2}$ is a solution of $\mathfrak{A} v=0$ such that $v\left(s_{1}\right)=v\left(s_{2}\right)=0$. By Lemma 4.1 then $v=0$ in the interval $\left(s_{1}, s_{2}\right)$, and, since $s_{1}$ and $s_{2}$ are arbitrary, $v=0$ in $N_{s} \cap N_{s}^{\prime}$. Thus there exists a linear relationship between $u_{1}, u_{2}$, and 1 . It follows that 1 was in the domain of the original (unextended) operator $\mathfrak{A}$. Furthermore, $u_{1}$ and $u_{2}$ being independent, there exists a linear combination $x=p_{1} u_{1}+p_{2} u_{2}$ which is independent of $\mathbf{1}$ and therefore strictly monotonic, say increasing.

To prove the lemma "in the small", suppose that the function $u$ satisfies $\mathfrak{A} u=0$ in some subinterval $I^{\prime} \subset N_{s} \cap N_{s}^{\prime}$. In this interval also $v=$ $u-p x-q \mathbf{1}$ satisfies $\mathfrak{A} v=0$, and the constants $p, q$ may be chosen so that $v\left(s_{1}\right)=v\left(s_{2}\right)$. It follows then from Lemma 4.1 that $v$ vanishes in $\left(s_{1}, s_{2}\right)$, and thus $u=p x+q 1$.

It remains to prove the lemma "in the large." For that purpose choose among the intervals $N_{s} \cap N_{s}^{\prime}$ a doubly infinite sequence, say $\cdots, I_{-1}, I_{0}$, $I_{1}, \cdots$, whose union covers $I$ and such that $I_{k}$ overlaps $I_{k-1}$ and $I_{k+1}$, but no other interval $I_{j}$. We have shown that in each $I_{k}$ there exists a strictly increasing function $x_{k}$ satisfying $\mathfrak{A} x_{k}=0$. In $I_{0}$ we put $x=x_{0}$. In the 
intersection $I_{0} \cap I_{1}$ we have $x=p_{1} x_{1}+q_{1} 1$, and we use this equation as a definition of $x$ throughout $I_{1}$. Then $\mathfrak{A} x=0$ in the union $I_{0} \cup I_{1}$, and $x$ is strictly increasing. Proceeding in this way by induction we construct a strictly increasing solution $x$ of $\mathfrak{A} x=0$ defined in the union $\bigcup_{i=-k}^{k} I_{i}$, and hence in $I$. Finally, if $\mathfrak{A} u=0$ in some interval $I^{\prime} \subset I$, then in each $I_{k} \cap I^{\prime}$ the function $u$ is of the form $u=p_{k} x+q_{k} 1$. Since $I_{k}$ and $I_{k+1}$ overlap, we have $p_{k}=p_{k+1}, q_{k}=q_{k+1}$, and the lemma is proved.

To complete the proof of Theorem 2.2 we have merely to observe that the conditions (3.1) of Theorem 3.1 hold in consequence of the preceding lemma.

\section{Proof of Theorem 2.1}

We shall again reduce this theorem to Theorem 3.1. As in the preceding section we suppose that $\mathfrak{U}$ is defined in the interval $I: s_{-}<s<s_{+}$and has the properties (1), (2), $\left(3^{\prime}\right)$. We denote by $s$ the function which at each point $s$ equals $s$.

LEmma 5.1. Suppose that in an interval $I_{0} \subset I$ one has $\mathfrak{A} \mathbf{s}=\beta \geqq 0$. If $f$ decreases and $\mathfrak{A} f>0$ in $I_{0}$, then the graph of $f$, as a function of $s$, is convex downward.

Proof. We refer to the proof of Lemma 3.1 replacing the scale parameter $x$ by $s$. We have then $\mathfrak{A} u=\mathfrak{A} f-p \beta$. All chords are inclined downward, whence $p<0$ and $\mathfrak{A} u>0$. Thus the argument of the original proof applies in the present case.

Lemma 5.2. Let $s_{0}$ be a regular point. A neighborhood $N$ of $s_{0}$ can be parametrized by a parameter $x$ (a continuous strictly increasing function of $s$ ) in such a way that whenever $\mathfrak{A} f$ is continuous in some subinterval of $N$, the one-sided derivatives $D_{x}^{+} f$ and $D_{x}^{-} f$ exist and satisfy the continuity conditions (2.4).

Proof. By Definition 2.2 of regular points we can find a neighborhood $N$ of $s_{0}$ in which there exist an increasing function $x$ and a decreasing function $v$ such that $\mathfrak{A} x>0$ and $\mathfrak{A} v>0$. These functions are strictly monotonic (see Lemma 2.2). We choose $x$ as a local parameter in $N$. In terms of it $N$ becomes the interval $x_{1}<x<x_{2}$, and in $N$ we have

$$
\mathfrak{A} \mathbf{x}=\beta \geqq 0, \quad \mathfrak{A} v>0, \quad v \downarrow \text {. }
$$

To prove the lemma it suffices to show that (5.1) implies the following: if $\mathfrak{A} f$ is continuous in the open interval $N^{\prime} \subset N$, then in any closed subinterval $N^{\prime \prime} \subset N^{\prime}$ the function $f$ admits a representation as the difference of two convex functions of $x$.

Let $N^{\prime}: x_{1}^{\prime}<x<x_{2}^{\prime}$ and $N^{\prime \prime}: x_{1}^{\prime \prime}<x<x_{2}^{\prime \prime}$. Since $\mathfrak{Y} v$ is strictly positive and $v$ strictly decreasing in $N$, we may choose the real parameter $t$ so large that for $F=f+t v$ we shall have $\mathfrak{X} F>0$ in $N^{\prime}$ and $F\left(x_{2}^{\prime}\right)<F\left(x_{2}^{\prime \prime}\right)$. It follows then that $F$ decreases in $N^{\prime \prime}$. In fact, if $x_{1}^{\prime}<x<x_{2}^{\prime \prime}<x_{2}^{\prime}$, then the maximum of $F$ in the interval $\left(x, x_{2}^{\prime}\right)$ cannot be attained at an interior point 
since there $\mathfrak{A} F>0$. This maximum is not attained at the right endpoint $x_{2}^{\prime}$ since $F\left(x_{2}^{\prime}\right)<F\left(x_{2}^{\prime \prime}\right)$. Hence the maximum is attained at the left endpoint $x$, and thus $F$ decreases in $\left(x_{1}^{\prime}, x_{2}^{\prime \prime}\right)$. By Lemma 5.1 both $F$ and $v$ are, as functions of $x$, convex, and since $f=F-t v$, the assertion is proved.

Completion of the proof. Using the notation of the last lemma define in $\mathrm{N}$ a new operator $\mathfrak{A}^{*}$ by

$$
\mathfrak{A} *_{f}=\mathfrak{A} f-\beta \cdot D_{x}^{-} f .
$$

This operator $\mathfrak{A}^{*}$ has again the characteristic properties (1), (2), (3'). The local character is obvious. That $\mathfrak{A}^{*}$ is nontrivial is seen from the fact that $v$ being decreasing we have $D_{x}^{-} v \leqq 0$ and hence $\mathfrak{A}^{*} v \geqq \mathfrak{A} v>0$. Finally, at a place of a local minimum of $f$ we have $D_{x}^{-} f \leqq 0$ and therefore $\mathfrak{Y} *_{f} \geqq \mathfrak{A} f \geqq 0$ for each $f$ in the local domain of $\mathfrak{A}^{*}$.

It is clear from the definition that $\mathfrak{Y}^{*} \mathrm{x}=0$, and Lemma 4.2 shows that either $\mathfrak{I}^{*} 1=0$ or we are free to supplement the definition of $\mathfrak{I}^{*}$ by setting $\mathfrak{A} * 1=0$. The conditions (3.1) of Theorem 3.1 are then satisfied, and we can apply this theorem to describe the structure of $\mathfrak{A}^{*}$. It is true that $\mathfrak{A}^{*} f$ need not be continuous since $D_{x}^{-} f$ may have jumps. However, in consequence of the continuity conditions (2.4) and the continuity of $\beta$, it is clear that for each $f$ in its domain $\mathfrak{A}^{*} f$ is continuous on the left, and that even the right limits exist at each point. The proof of Theorem 3.1 applies under these circumstances without change and shows that there exists a function $\mu$ such that $\mathfrak{U}^{*} f=D_{\mu} D_{x} f$. If then in $N$ we define the operator

$$
\overline{\mathfrak{A}}=D_{\mu} D_{x}+\beta D_{x}^{-},
$$

we see that $\overline{\mathfrak{A}}$ is, in $N$, an extension of $\mathfrak{A}$.

We wish to apply Theorem 3.1 to reduce $\overline{\mathfrak{A}}$ to the canonical form

$$
\overline{\mathfrak{A}}=D_{m} D_{u} \text {. }
$$

For that purpose we require in $N$ a strictly increasing function $u$ satisfying $\overline{\mathfrak{A}} u=0$. Introducing $u$ as local parameter in $N$ we get (5.4) as an immediate consequence of Theorem 3.1. To construct the desired function $u$, we use the familiar method of successive approximations to show that the differential equation

$$
D_{\mu}^{-} v+\beta v=0
$$

admits, in $N$, of a positive solution $v$. (This $v$ is continuous except, perhaps, at points of discontinuity of $\mu$.) Clearly $u=\int v d x$ is a strictly increasing solution of $\overline{\mathfrak{A}} u=0$ (defined up to a linear transformation $u \rightarrow p u+q \mathbf{1}$ ).

We have thus proved Theorem 2.1 "in the small", namely that to each regular point $s_{0} \in I$ there corresponds a neighborhood $N$ in which $\mathfrak{A} f=D_{m} D_{u} f$ for each $f$ in the domain of $\mathfrak{A}$. To show that a similar representation holds "in the large" throughout $I$ we have merely to repeat the concluding argument of the last section. 


\section{Singular points}

We proceed to show that at irregular points $\mathfrak{A}$ degenerates into a first order differential operator.

Theorem 6.1. Suppose that in the interval $I: s_{-}<s<s_{+}$the operator $\mathfrak{A}$ has the properties (1), (2), ( $\left.3^{\prime}\right)$.

Then there exists in I a strictly increasing continuous function $x$ such that for each nonregular point $s \in I$ either

$$
\begin{gathered}
\mathfrak{A} f(s)=D_{x}^{+} f(s) \text { for all } f \in D(\mathfrak{A}, s) \\
\mathfrak{U} f(s)=-D_{x}^{+} f(s) \text { for all } f \in D(\mathfrak{A}, s) .
\end{gathered}
$$

This theorem was formulated so as to reveal the salient features in the simplest manner. Actually the proof will automatically provide further information embodied in the following

Amplification. If $s$ is an irregular point, then exactly one of the following five mutually exclusive situations prevails:

For $f \in D(\mathfrak{A}, s)$ the relation $\mathfrak{A} f(s)>0$ implies that:

(i) $f$ is strictly increasing near $s$;

(ii) $f$ is either increasing or has a strict local minimum at $s$, each possibility actually occurring for some $f$;

(iii) $f$ has a strict local minimum at $s$;

(iv)-(v) same as (i), (ii) with "increasing" replaced by "decreasing."

In the cases (i) and (v) we can replace (6.1)-(6.2) by

$$
\mathfrak{A} f(s)= \pm D_{x} f(s),
$$

where $D_{x}$ is a two-sided derivative. In the case (iii)

$$
\mathfrak{U} f(s)=D_{x}^{+} f(s)=-D_{x}^{-} f(s) .
$$

Proof "in the small." We prove that to each singular point $s_{0} \in I$ there corresponds a neighborhood $N$ such that Theorem 6.1 and its amplification are true when $I$ is replaced by $N$.

The fact that there exist only the five types of singular points enumerated above is an immediate consequence of Lemma 2.2 .

Let $s$ be a singular point. For reasons of symmetry it suffices to consider the case where $\mathfrak{A} f\left(s_{0}\right)>0$ implies that $f$ is not strictly decreasing (types (i)(iii) above). In view of property (2) we may choose a neighborhood of $s_{0}$ in which there exists a function $v$ such that

$$
\mathfrak{A} v=\beta>0 .
$$

Then $v$ is strictly increasing in a right neighborhood $s_{0} \leqq s<s_{0}+\varepsilon$ and strictly monotone in the left neighborhood $s_{0}-\varepsilon<s \leqq s_{0}$. We choose $\varepsilon$ so small that (6.5) holds in the neighborhood $N: s_{0}-\varepsilon<s<s_{0}+\varepsilon$. 
We next prove that

$$
\mathfrak{A} f\left(s_{0}\right)=\beta\left(s_{0}\right) D_{v}^{+} f\left(s_{0}\right)
$$

for each $f \in D\left(\mathfrak{A}, s_{0}\right)$. For this purpose we introduce the function defined in $N$ by

$$
F_{t}=\beta\left(s_{0}\right) f-t v
$$

depending on the real parameter $t$. Clearly $F_{t} \in D\left(\mathfrak{A}, s_{0}\right)$. If $t<\mathfrak{A} f\left(s_{0}\right)$, we have $\mathfrak{A} F_{t}\left(s_{0}\right)>0$ implying that $F_{t}$ increases in a right neighborhood of $s_{0}$, i.e. that $F_{t}(s)>F_{t}\left(s_{0}\right)$ for $s_{0}<s<s_{0}+\delta$. Then

$$
\beta\left(s_{0}\right)\left\{f(s)-f\left(s_{0}\right)\right\}>t\left\{v(s)-v\left(s_{0}\right)\right\},
$$

or

$$
\beta\left(s_{0}\right) \liminf _{s \downarrow s_{0}} \frac{f(s)-f\left(s_{0}\right)}{v(s)-v\left(s_{0}\right)} \geqq \mathfrak{A} f\left(s_{0}\right) .
$$

For $t>\mathfrak{A} f\left(s_{0}\right)$ the same argument yields the reversed inequality for the limit superior, and thus (6.6) holds.

In the interval $N$ we now introduce the strictly increasing function $x$ defined by

$$
x(s)=\int_{s_{0}}^{s} \frac{|d v|}{\beta}+\text { const. }
$$

Then $D_{x}^{+} f\left(s_{0}\right)=\beta\left(s_{0}\right) D_{v}^{+} f\left(s_{0}\right)$, and (6.1) is proved at the point $s_{0}$.

More precisely, if $s_{0}$ is of the type (i), then $v$ is strictly increasing, and $\mathfrak{A} F_{t}>0$ implies that also $F_{t}$ increases near $s_{0}$. In this case the situation is perfectly symmetric for right and left neighborhoods, and we have not only (6.6) but also $\mathfrak{X} f\left(s_{0}\right)=\beta\left(s_{0}\right) D_{0}^{-} f\left(s_{0}\right)$ and hence (6.3) with the positive sign. Similarly, if $s_{0}$ is of type (iii), $v$ has a minimum at $s_{0}$, and $D_{x}^{-} f\left(s_{0}\right)=$ $-\beta\left(s_{0}\right) D_{v}^{-} f\left(s_{0}\right)$. In this case (6.4) holds. Finally, when $s_{0}$ is of type (ii), then $v$ may be chosen strictly increasing, but it is impossible to strengthen (6.1) by making a generally valid statement about the left derivative (which, however, exists in any case; see example (c), Section 10).

Finally, note that when $s \in N$ is any other singular point, the argument applies with the same $v$ and $x$, and hence the theorem is proved for the neighborhood $N$.

Proof "in the large." (This part of the theorem is of minor interest and is proved for completeness only.) We proceed in two steps. First consider the special case where all points of $I$ are singular. We can cover $I$ by denumerably many open intervals $\cdots, N_{-1}, N_{0}, N_{1}, \cdots$ such that in $N_{k}$ there exists a strictly increasing function $x_{k}$ in terms of which $\overline{\mathfrak{A}} f= \pm D_{x_{k}}^{ \pm} f$ as indicated in the theorem. Removing, if necessary, superfluous intervals, we suppose that $N_{k}$ overlaps $N_{k-1}$ and $N_{k+1}$, but no other $N_{j}$. Clearly $x_{k}$ is determined uniquely up to an additive constant, and we can choose these additive constants in $N_{0}, N_{1}, N_{-1}, N_{2}, N_{-2}, \cdots$ successively in such a way that $x_{k}$ and $x_{k+1}$ coincide in the overlap $N_{k} \cap N_{k+1}$. Then each $x_{k}$ is the restriction 
of an increasing function $x$ defined throughout $I$, and for it Theorem 6.1 holds true.

Next, consider the general case where $I$ contains both regular and irregular points. If an interval $I_{0} \subset I$ contains only irregular points, then we have shown that there exists an interval $N \supset I_{0}$ in which the theorem is true. In other words, to each irregular $s \in I$ we can find a neighborhood $N$ such that the theorem is valid for $N$ and the endpoints of $N$ are regular points (or endpoints of the basic interval $I$ ). By definition the regular points form an open set, and hence the set $S$ of singular points is closed in $I$. We can therefore cover $S$ by denumerably many intervals $N_{k}$ such that in $N_{k}$ the theorem is true for a certain function $x_{k}$ and the endpoints of $N_{k}$ are regular. We may suppose the $N_{k}$ nonoverlapping and ordered, i.e. $N_{k}$ lies entirely to the left of $N_{k+1}$. Then $I-U N_{k}$ consists of denumerably many closed intervals (containing only regular points), and Theorem 6.1 has no bearing on them: In them $x$ may be defined in a perfectly arbitrary manner. Thus we may in $N_{k}$ define $x=x_{k}+c_{k}$ and dispose of the constants $c_{1}, c_{-1}, c_{2}, \cdots$ successively in such a manner that $x$ will be increasing in $U N_{k}$. In the complementary set we define $x$ by linear interpolation and the theorem holds for the function $x$ thus defined.

\section{The natural scale}

We have seen that for operators $\mathfrak{A}$ with the strong minimum property $\left(3^{\prime}\right)$ an interval $I$ not containing first order points may be referred to a parameter $x$ in terms of which $\mathfrak{A}$ assumes a particularly simple form. This result will now be extended to operators enjoying only the weak minimum property (3). It will be shown that the equation $\mathfrak{A} u=0$ can have at most two independent solutions (provided independence is defined as in footnote 3 ; cf. example (c) of Section 10). At present we shall assume that, at least locally, there actually exist two such solutions. Later on we shall remove this restriction.

Theorem 7.1. Suppose that in the interval $I: s_{-}<s<s_{+}$the operator $\mathfrak{U}$ has the properties (1), (2), (3), and assume that to each $s \in I$ there corresponds a neighborhood $N$ in which the equation $\mathfrak{A} u=0$ possesses two independent positive solutions.

Then there exist two independent functions $\phi_{i}$ defined in $I$ and satisfying throughout $I$ the equations $\mathfrak{N} \phi_{i}=0$. If $\mathfrak{A} u=0$ in some interval $I_{0} \subset I$, then

$$
u=p_{1} \phi_{1}+p_{2} \phi_{2}
$$

where the $p_{i}$ are constants.

The interval I may be parametrized by a variable $x$ (a strictly increasing continuous function of $s$ ) in such a way that for two arbitrary functions $\phi$ and $\psi$ satisfying the equations $\mathfrak{N} \phi=0$ and $\mathfrak{A} \psi=0$ the ratio $\psi / \phi$ is differentiable with respect to $x$ in any interval $I_{0}$ not containing a zero of $\phi$. Furthermore

$$
\phi^{2}(\psi / \phi)^{\prime}=\text { const. }
$$


or, equivalently,

$$
\psi=k_{1} \phi \int \frac{d x}{\phi^{2}}+k_{2} \phi
$$

where $k_{i}=$ const. The parameter $x$ is defined uniquely up to a trivial linear transformation $x \rightarrow p x+q 1$.

The proof will be based on the following

Lemma 7.1. Let $\mathfrak{A}$ have the properties (1), (2), (3), and suppose that in the interval $I$ we have $\phi>0$ and $\mathfrak{N} \phi=0$.

Define an operator $\mathfrak{2}^{*}$ by

$$
\mathfrak{A} *_{f}=\mathfrak{A}(f \phi) .
$$

Then $\mathfrak{Y}^{*}$ has the properties (1), (2), (3').

Proof. Only the strong minimum property $\left(3^{\prime}\right)$ requires comment. The domain of $\mathfrak{A}^{*}$ contains all $f$ such that $f \phi$ is in the domain of $\mathfrak{A}$. Let $f$ be such a function with a local minimum at the point $s_{0}$. The function $F$ defined by

$$
F(s)=\left\{f(s)-f\left(s_{0}\right)\right\} \phi(s)
$$

vanishes at $s_{0}$ and is nonnegative in a neighborhood of $s_{0}$. Clearly $F \in D\left(\mathfrak{A}, s_{0}\right)$, and by the property (3) we have $\mathfrak{A} F\left(s_{0}\right) \geqq 0$. Now

$$
\mathfrak{U} *_{f}=\mathfrak{A} F+f\left(s_{0}\right) \mathfrak{A} \phi=\mathfrak{U} F,
$$

and therefore $\mathfrak{U}^{*}$ has the strong minimum property $\left(3^{\prime}\right)$.

Proof of Theorem 7.1. Let $N$ be a neighborhood of $s_{0} \in I$ such that in $N$ we have $u_{i}>0$ and $\mathfrak{A} u_{i}=0$, where $u_{1}$ and $u_{2}$ are linearly independent. In $N$ we define $\mathfrak{A}^{*}$ as in (7.3) putting $\phi=u_{1}$. Then $\mathfrak{A}^{*} \mathfrak{1}=0$, and $\mathfrak{A}^{*}\left(u_{2} / u_{1}\right)=0$. Thus $\mathfrak{I}^{*} z=0$ has two linearly independent solutions, and Theorem 2.2 applies to $\mathfrak{A}^{*}$. It implies that the ratio $u_{2} / u_{1}$ is strictly monotonic, in fact

$$
u_{2}=k_{1} \xi u_{1}+k_{2} u_{1}
$$

where $\xi$ is the natural scale for $\mathfrak{A}^{*}$ in the interval $N$ and $k_{i}=$ const.

Unfortunately this $\xi$ depends on the arbitrary choice of the two solutions $u_{i}$, and we require a parameter $x$ essentially independent of this choice. A very elementary calculation will show that the function $x$ defined by

$$
x=\int_{s_{0}}^{s} u_{1}^{2} d \frac{u_{2}}{u_{1}}
$$

has this property. More precisely: if $v_{i}>0$ and $\mathfrak{A} v_{i}=0$, then the functions $w_{i}=v_{i} / \phi$ satisfy $\mathfrak{P}^{*} w_{i}=0$ and are in consequence of Theorem 2.2 linear combinations of $\xi$ and 1 . It follows that

$$
v_{1}=\alpha_{1} u_{1}+\alpha_{2} u_{2}, \quad v_{2}=\beta_{1} u_{1}+\beta_{2} u_{2},
$$


where the $\alpha_{i}$ and $\beta_{i}$ are constants. Now clearly

$$
\int_{s_{0}}^{s} v_{1}^{2} d \frac{v_{2}}{v_{1}}=\left(\beta_{2} \alpha_{1}-\alpha_{2} \beta_{1}\right) x
$$

We have thus proved the theorem "in the small," namely when $I$ is replaced by the interval $N$. A simple repetition of the argument used at the end of Section 4 shows then the validity of the theorem "in the large."

\section{The first representation theorem}

TheOREm 8.1. Under the assumptions of Theorem 7.1 we have, whenever $\mathfrak{U} f$ exists,

$$
\mathfrak{A} f=\frac{1}{\phi} D_{m}\left(\phi^{2} D_{x} \frac{f}{\phi}\right)=\frac{1}{\psi} D_{m}\left(\psi^{2} D_{x} \frac{f}{\psi}\right) .
$$

Here $m$ is a strictly increasing right-continuous function defined in $I$. For a fixed choice of the scale parameter $x$ the "canonical measure" $m$ is determined uniquely up to an irrelevant additive constant.

Proof. It suffices to prove the assertion for the fixed neighborhood $N$ introduced in the proof of Theorem 7.1. In consequence of Theorem 2.2 we have in $N$ the representation

$$
\mathfrak{U}^{*} f=D_{\mu} D_{\xi} f
$$

with an appropriate monotone $\mu$. The scale parameter $\xi$ depends on an arbitrary multiplicative constant, and we may dispose of it in such a way that in (7.6) we have $k_{1}=1$. A comparison of (7.6) and (7.7) then shows that

$$
D_{\xi}=u_{1}^{2} D_{x},
$$

and hence (putting as before $u_{1}=\phi$ )

$$
\mathfrak{A} f=\mathfrak{A} * \frac{f}{\phi} D_{\mu}\left(\phi^{2} D_{x} \frac{f}{\phi}\right) .
$$

${ }^{5}$ At a zero of $\psi$ the last term in (8.1) is senseless, and the corresponding statement in (8.1) should be interpreted as empty. Since $\phi$ and $\psi$ cannot vanish at the same point, we have in (8.1) always a valid representation for $\mathfrak{A}$. The formal definition of the right side in (8.1) is in all respects analogous to Definition 2.1.

Added in proof. The operator $\Omega$ defined by $\Omega f=D_{m} D_{x} f+c f$ is always reducible to the canonical form (8.1), but the converse is true only if $\phi$ is sufficiently regular. Thus if $\phi^{\prime}=n$ exists and is of bounded variation (not necessarily continuous), then the integrated version of (8.1) reduces to

$$
\int_{x_{1}+}^{x_{2}-} \mathfrak{A} f \cdot d m=f^{-}\left(x_{2}\right)-f^{+}\left(x_{1}\right)-\int_{x_{1}+}^{x_{2}-} \frac{1}{\phi} f d n .
$$

If $n$ is absolutely continuous with respect to $m$, then $\left(^{*}\right)$ is equivalent to $\mathscr{A} f=$ $D_{m} D_{x} f+c f$ with $c=\phi^{-1} d n / d m$. The case $c \leqq 0$ corresponds in the most general case to a conyex $\phi$. For details see [2]. 
Here $\mu$ still depends on the choice of the solution $\phi$; to prove (8.1) we have to show that

$$
m=\int \frac{d \mu}{\phi}
$$

is independent of this choice, and thus intrinsically connected with $\mathfrak{A}$. For a particular choice of $\phi>0$ let us define $m$ by (8.5). Then the first formula in (8.1) holds, and we have to prove that for arbitrary $s_{1}<s_{2} \epsilon N$ we have

$$
\int_{s_{1}}^{s_{2}} \frac{\psi}{\phi} d\left(\phi^{2} D_{x} \frac{f}{\phi}\right)=\int_{s_{1}}^{s_{2}} d\left(\psi^{2} D_{x} \frac{f}{\psi}\right)=\left.\psi^{2} D_{x} \frac{f}{\psi}\right|_{s_{1}} ^{s_{2}} \text {. }
$$

The truth of (8.6) follows simply by using integration by parts remembering that $\phi$ and $\psi$ are related by $(7.1)$.

\section{The general case}

We turn to an investigation of operators $\mathfrak{A}$ with the properties (1), (2), (3) without assuming the existence of solutions of $\mathfrak{H} u=0$. We shall show that a representation of the form (8.1) holds except when the interval contains singular points. These can be characterized intrinsically by analogy with Definition 2.2, but for simplicity of formulations we shall be satisfied with the following purely formal

Definition 9.1. Let $\mathfrak{A}$ be defined in the interval $I: s_{-}<s<s_{+}$and have properties (1), (2), (3). A point $s_{0} \in I$ is called of zero order if

$$
\mathfrak{A} f\left(s_{0}\right)=k f\left(s_{0}\right) \text { for each } f \in D\left(\mathfrak{A}, s_{0}\right) \text {. }
$$

$A$ point $s_{0} \epsilon I$ is of first order if there exist in a neighborhood $N$ of $s_{0}$ a strictly increasing continuous function $y$ and a continuous function $\lambda$ such that

$$
\mathfrak{P} f\left(s_{0}\right)= \pm D_{y}^{ \pm}(f \lambda)\left(s_{0}\right) \text { for each } f \in D\left(\mathfrak{A}, s_{0}\right) \text {. }
$$

A point is regular if it is neither of zero nor of first order.

With this convention we have

Theonem 9.1. Suppose that $\mathfrak{A}$ is defined in the interval $I$ and has the properties (1), (2), (3). Suppose furthermore that all points of I are regular.

Then $\mathfrak{A}$ can be extended to an operator as described in Theorem 8.1. (In other words, a scale parameter $x$ and a canonical measure $m$ can be introduced so that for each $f$ in the domain of $\mathfrak{H}$ formula (8.1) holds, and $\phi, \psi$ stand in the relation $(7.1)$.)

Proof. It obviously suffices to show that the assertion is true for some neighborhood of an arbitrary point $s_{0} \epsilon I$. Let $N$ be an interval around $s_{0}$ in which there exists a function $w$ such that $w>0$ and $\mathfrak{A} w \neq 0$. The existence of such an interval is guaranteed by property (2). In $N$ we define (by anal- 
ogy with (7.3)) an operator $\mathfrak{U}^{*}$ by

$$
\mathfrak{A}^{*} f=\mathfrak{A}(f w)-f \cdot \mathfrak{A} w .
$$

Obviously $\mathfrak{A} *$ is of local character and has the property (3). Now $\mathfrak{A} * 1=0$, and therefore $\mathfrak{I}^{*}$ enjoys even the strong minimum property $\left(3^{\prime}\right)$. We have now to distinguish three cases:

(a) At some point $s \in N$ the operator $\mathfrak{A}^{*}$ is trivial, that is, $\mathfrak{A}^{*}$ does not have the property (2). Since $\mathfrak{H}^{*} \mathfrak{1}=0$, this is equivalent to saying that $\mathfrak{U}^{*} f(s)=0$ for each $f \in D\left(\mathfrak{Y}^{*}, s\right)$, or that

$$
\mathfrak{A} f(s)=k f(s) \quad \text { where } \quad k=w^{-1}(s) \cdot \mathfrak{A} w(s)
$$

for each $f \in D(\mathfrak{A}, s)$. In this case $s$ is a point of zero order.

In the absence of zero order points $\mathfrak{I}^{*}$ has the properties (1), (2), (3'), and the theorems of Sections 2 and 6 apply. The next possibility is then:

(b) The operator $\mathfrak{A}^{*}$ has properties (1), (2), ( $\left.3^{\prime}\right)$, but some point $s \in N$ is a first order point. In this case there exists a strictly increasing continuous function $\xi$ such that either

$$
\mathfrak{I}^{*} f(s)=D_{\xi}^{+} f(s) \text { for each } f \in D\left(\mathfrak{Y}^{*}, s\right),
$$

or an analogous equation with $D_{\xi}^{+}$replaced by $-D_{\xi}^{+}$or $\pm D_{\xi}^{-}$holds. Now (9.5) is equivalent to saying that for each $f \in D(\mathfrak{A}, s)$

$$
\mathfrak{A} f=D_{\xi}^{+} \frac{f}{w}+\frac{f}{w} \cdot \mathfrak{A} w
$$

holds at the particular point $s$. Put

$$
W=\exp \left\{\int(\mathfrak{A} w) \cdot d \xi\right\}
$$

and

$$
y=\int W d \xi
$$

Then (9.6) reduces to

$$
\mathfrak{A} f=D_{y}^{+}\left(\frac{f}{w} W\right)
$$

and thus $s$ is a first order point.

(c) If $N$ contains neither zero- nor first-order points, then $\mathfrak{H}^{*}$ has the properties (1), (2), $\left(3^{\prime}\right)$, and all points are regular. By Theorem 2.1 in this case for each $f \in D\left(\mathfrak{A}^{*}, N\right)$

$$
\mathfrak{U}^{*} f=D_{\mu} D_{\xi} f
$$

for some monotone functions $\xi$ and $\mu$. Formula (9.3) now shows that in this case the operator $\overline{\mathfrak{X}}$ defined in $N$ by

$$
\overline{\mathfrak{A}} f=D_{\mu} D_{\xi} \frac{f}{w}-\frac{f}{w} c, \quad c=w^{-1} \cdot \mathfrak{A} w
$$

is an extension of $\mathfrak{A}$ (i.e. $\mathfrak{A} f=\overline{\mathfrak{A}} f$ whenever the left side is continuous). 
The argument of Lemma 2.1 shows directly that $\overline{\mathfrak{A}}$ has the minimum property (3), and it is obvious that $\mathfrak{A}$ has the properties (1), (2). The classical method of successive approximations proves the existence of a solution of the differential equation $\overline{\mathfrak{A}} u=0$ such that $u\left(s_{0}\right)=\alpha$ and $D_{\xi}^{+} u\left(s_{0}\right)=\beta$, where $\alpha$ and $\beta$ are arbitrary constants. Therefore, in a neighborhood $N^{\prime}$ of $s_{0}$ there exist two positive independent solutions $u_{i}$ of $\overline{\mathfrak{A}} u=0$, and in $N^{\prime}$ Theorem 8.1 applies to $\overline{\mathfrak{A}}$. Thus to each point $s_{0} \epsilon I$ there corresponds a neighborhood $N^{\prime}$ in which $\mathfrak{A}$ has an extension $\overrightarrow{\mathfrak{A}}$ of the form described in Theorem 8.1, and Theorem 9.1 is proved.

\section{Examples}

(a) Removable singularities. Let $\mathfrak{A}$ be defined only on the set of polynomials in $x^{2}$ (even polynomials), and for each such polynomial $\mathfrak{A} p=p^{\prime \prime}$. Here the origin is a first order point of type (iii) in the classification of Section 6 : if $p^{\prime \prime}(0)>0$, then $p$ has a strict local minimum at the origin.

Obviously the second derivative $D_{x}^{2}$ is an extension of $\mathfrak{A}$ for which all points are regular. In this sense the singularity of the origin for $\mathfrak{A}$ is due to the fact that $\mathfrak{A}$ is defined on too small a domain. However, one should not jump to the hasty conclusion that $D_{x}^{2}$ is the "natural" or unique extension of our $\mathfrak{A}$. The next example shows a different extension of $\mathfrak{A}$ which appears in diffusion theory and for which the origin is a first order point of type (ii).

(b) An isolated first order point. Let $\mathfrak{Q}$ be defined in $(-\infty, \infty)$ as follows: for $x \neq 0$ we put $\mathfrak{A} f(x)=f^{\prime \prime}(x)$. For $f$ to be in the domain of $\mathfrak{A}$ at the origin we require that $f$ be continuous,

$$
f^{+}(0)=0, \quad \lim _{x \rightarrow 0} f^{\prime \prime}(x)=f^{++}(0)=\mathfrak{U} f(0) .
$$

The operator $\mathfrak{A}$ so defined has applications in diffusion theory ${ }^{6}$ and shares many essential properties of the ordinary second derivative. For example, it is easily shown that for a bounded continuous $f$ the equation

$$
\lambda F-\mathfrak{A} F=f
$$$$
\lambda>0
$$

has exactly one bounded solution $F$.

Despite their strange appearance equations (10.1) state simply that the origin is a first order point. If we define a strictly monotonic function by $\xi(x)=\frac{1}{2} x^{2}$ for $x \geqq 0$ (and arbitrarily for $x<0$ ), then (10.1) is equivalent to $\mathfrak{A} f(0)=D_{\xi}^{+} f(0)$. Thus the origin is of type (ii). Note that this $\mathfrak{A}$ extends the operator of example (a).

${ }^{6}$ Equation (10.2) is the Laplace transform of the diffusion equation for the following situation. In the half interval $x<0$ we have an ordinary homogeneous diffusion with an absorbing barrier at the origin; in $x>0$ we have an ordinary diffusion with a reflecting barrier at the origin. If, starting from $x<0$, the particle reaches the origin, it immediately penetrates into the right half-line. Ordinarily (10.1) would be interpreted as a "transition condition" for two separate diffusion processes. 
(c) Linear independence. Let $\mathfrak{A}$ be the operator of example (b), and put $\mathfrak{I}^{*}=\mathfrak{U}-\lambda^{2}$ where $\lambda$ is a constant. Clearly $\mathfrak{I}^{*}$ has the properties (1), (2), (3), and the origin is a first order point for $\mathfrak{A}^{*}$. Jefine $u_{i}$ by

$$
u_{1}(x)= \begin{cases}\exp (\lambda x) & \text { for } x \leqq 0 \\ \cosh (\lambda x) & \text { for } x \geqq 0\end{cases}
$$

and

$$
u_{2}(x)= \begin{cases}\sinh (\lambda x) & \text { for } x \leqq 0 \\ 0 & \text { for } x \geqq 0 .\end{cases}
$$

Then $\mathfrak{A}^{*} u_{i}=0$. There exists no linear combination $p_{1} u_{1}+p_{2} u_{2}$ which vanishes identically in a neighborhood of 0 , but nevertheless $u_{1}$ and $u_{2}$ are not independent in the sense of our definition of footnote 3.

\section{REFERENCES}

1. W. Feluen, On second order differential operators, Ann. of Math. (2), vol. 61 (1955), pp. 90-105.

2. - , Generalized second order differential operators and their lateral conditions, Illinois J. Math., vol. 1 (1957), pp. 459-504.

3. H. P. MCKean, JR., Elementary solutions for certain parabolic partial differential equations, Trans. Amer. Math. Soc., vol. 82 (1956), pp. 519-548.

4. Added in proof. For applications to the theory of the vibrating string see a forthcoming note in the Lévy testimonial volume of the Publications de l'Institut Statistique de l'Université de Paris.

\section{Princeton University}

Princeron, New Jersey 